\title{
Association between Clinical Characteristics and Quality of Life in Older People with Stroke at Hospital Discharge
}

\author{
Saisamorn Chaleoykitti1 ${ }^{*}$, Werayuth Srithumsuk ${ }^{2}$, Saitip Jaipong ${ }^{3}$, \\ Pinthusorn Pattayakorn ${ }^{4,5}$, Kattiya Podimuang ${ }^{6}$ \\ ${ }^{1}$ Royal Thai Army Nursing College, Bangkok, Thailand \\ ${ }^{2}$ Faculty of Nursing Science, Phetchaburi Rajabhat University, Phetchaburi, Thailand \\ ${ }^{3}$ Stroke Unit, Phrachomklao Hospital, Phetchaburi, Thailand \\ ${ }^{4}$ Department of Nursing, College of Natural Science, California State University San Bernardino, California, USA \\ ${ }^{5}$ Stroke Unit, Eisenhower Health, Rancho Mirage, California, USA \\ ${ }^{6}$ Annenberg 3 South Telemetry Unit, Eisenhower Health, Rancho Mirage, California, USA \\ Email: *saisamorncha2006@hotmail.com
}

How to cite this paper: Chaleoykitti, S., Srithumsuk, W., Jaipong, S., Pattayakorn, P. and Podimuang, K. (2020) Association between Clinical Characteristics and Quality of Life in Older People with Stroke at Hospital Discharge. Advances in Aging Research, 9, 67-76.

https://doi.org/10.4236/aar.2020.94006

Received: June 15, 2020

Accepted: July 14, 2020

Published: July 17, 2020

Copyright $\odot 2020$ by author(s) and Scientific Research Publishing Inc. This work is licensed under the Creative Commons Attribution International License (CC BY 4.0).

http://creativecommons.org/licenses/by/4.0/

\begin{abstract}
Objective: The number of people with stroke increases worldwide. The stroke survivors live with disabilities and those influence their quality of life (QOL). This study was aimed to investigate the association between clinical characteristics and QOL of the older people with stroke at discharge from the hospital. Methods: This is a cross-sectional study. The participants were 113 stroke survivors aged 60 years and older admitted to the stroke unit. Quality of life was the study's outcome which measured by using the abbreviated version of the World Health Organization Quality of Life (WHOQOL-BREF). Primary clinical characteristics were measured by the National Institute of Health Stroke Scale (NIHSS), Barthel Index (BI), and Modified Rankin Scale (mRS). Potential confounding factors were age, sex, education levels, marital status, current occupation, and comorbidity (hypertension, diabetes mellitus, dyslipidemia, and heart disease). Multiple linear regression was used for data analysis. Results: The main effects of clinical outcomes were high BI Score that had a significant difference association with QOL $(\beta=0.312,95 \% \mathrm{CI}=0.042,0.296$, $P=0.009)$, lower mRS score also had significant difference association with QOL $(\beta=-0.371,95 \% \mathrm{CI}=-5.394,-1.162, P=0.003)$ after all adjusting. Additional risk factor in this study was marital status (currently married) ( $\beta=$ $0.155,95 \% \mathrm{CI}=0.226,8.666, P=0.039$ ). Conclusion: Low function status and severe stroke disability as the clinical characteristics were associated with QOL in older people with stroke at hospital discharge. An additional factor was marital status (currently married).
\end{abstract}




\section{Keywords}

Functional Status, Older People, Quality of Life, Stroke, Stroke Disability

\section{Introduction}

Recently, stroke is the second leading cause of death and DALYs worldwide [1]. With an increase in number of older people in Thailand, a stroke occurs at any age, but it is predominantly found in older people. The average age of ischemic stroke patients in Thailand is approximately 65 years old, according to the record of Stroke in Thailand [2]. Stroke patients face the problems of physical, psychological, social and economic burdens. The patients' health-related quality of life (QOL) was found as low level [3] [4]. Some stroke survivors stated that their QOL would consider as being worse than death after having a stroke [5]. Therefore, understanding of the nature, severity of the symptoms of stroke, and the critical health consequences is needed. To delineate areas, it is important to assess how changes in physical, social, cognitive, and community participation affect QOL over time [6]. Additionally, a framework for designing future intervention models for stroke survivors needs to be developed to enhance their QOL [7].

Stroke survivors live with a new life challenge which is disabilities. In order to achieve functional recovery, patients with physical and/or mental sequelae need extensive rehabilitation. In addition, family, community, and social reintegration, as well as maintaining the level of rehabilitation are vital to help these stroke patients achieve a successful QOL [6]. Quality of life studies are crucial for evaluating the effect of stroke survivors' life on society. Literature reviews have shown that stroke affected various domains of quality of life, thereby affecting functionality [8] [9] [10]. However, most of the previous studies of QOL in stroke patients focused on physical, psychological, social, and cognitive function [11] [12] [13]. The period of QOL assessment of these studies focused on post-stroke from 2 weeks to 1 year in any age group. Unfortunately, only a few studies identified the stroke outcome factors associated with QOL, such as stroke severity, disability, and activity daily living in stroke survivors. The assessment of QOL during hospitalization is crucial because it will affect long-term stroke survivors' outcomes. Moreover, the study that focuses on the QOL of older adults with stroke is critically needed because the unique needs of care of this population may differ from other ages. This study aimed to investigate the association between clinical characteristics of stroke and quality of life in older people with stroke at discharge.

\section{Methods}

\subsection{Participants}

This cross-sectional study included patients hospitalized between November 2019 and March 2020 at the stroke unit in a government hospital in Phetchaburi province, Thailand. Inclusion criteria were: 1) age 60 and over 2) diagnosed with 
ischemic stroke or intracerebral hemorrhage, confirmed by brain imaging with the clinical presentation of stroke; 3 ) capable of cognitive communication; and 4) willingness to participate in the study. Exclusion criteria were: 1) severe physical conditions that endangered life or interfered with stroke recovery; 2) communication difficulties due to dysphasia or dysarthria that precluded informed consent and completion of the questionnaire; and 3) any of the comorbid neuropsychiatric conditions: dementia, Parkinson's disease, brain tumor, epilepsy, psychoses, or alcohol or substance dependence.

A total of 113 patients with stroke agreed to participate in the study and signed informed consent forms. The data were collected by using a structured questionnaire via personal interviews done by the researchers on the day of discharge. Clinical data were obtained from the medical records of the patients.

\subsection{Variables}

\subsubsection{Quality of Life}

QOL was measured using the abbreviated version of the World Health Organization Quality of Life (WHOQOL-BREF). WHOQOL-BREF tests domains related to physical factors, psychological factors, social participation, and the context of the environment. The patients were required to rate their QOL in the past two weeks. The item scores ranged from 1 to 5 , the total scores also ranged from 26 - 130, with a higher score indicating a better QOL. BREF was formally standardized in the Thai language version [14].

\subsubsection{Clinical Characteristics}

Clinical characteristics measurements performed by a physician at the discharge date and data were recorded from the medical records of the patients including National Institute of Health Stroke Scale (NIHSS), Barthel Index (BI) and Modified Rankin Scale (mRS). The NIHSS is a standardized assessment instrument that offers a quantitative measure of neurological impairment related to stroke. The scale consists of 15 items, including testing of consciousness level, selected cranial nerves, motor, sensory, the activity of the cerebellar, language, and inattention. In particular, a score of 0 usually indicates a normal function for each item. A score within the range of 1 to 4 indicates the level of impairment, with a higher score indicating the higher severity level of impairment. The total score is calculated by summing the individual item scores, with the possible maximum score being 42 and a minimum score is 0 [15].

The Activity Daily Living (ADL) was calculated by using the Barthel Index (BI) [16]. BI scores range from 0 (a dependent state) to 100 (a fully independent state), so higher scores indicate better performance in ADL. The Modified Rankin Scale (mRS) provides an assessment of the degree of disability, ranging from 0 to 6; higher score indicates higher level of disability [17].

\subsubsection{Sociodemographic Characteristics}

Data on age, sex, education levels, marital status, and current occupation were obtained from participants or caregivers, as appropriate. Analyses employed the 
following categories: age (years of age), sex (male vs. female), education (uneducated, primary school, secondary school, high school, diploma, Bachelor's degree, and master's degree and higher), marital status (currently married vs. currently unmarried), and occupation (employed vs. unemployed).

\subsubsection{Comorbidity}

The common comorbidity in stroke patients in this study included hypertension (HT), diabetes mellitus (DM), dyslipidemia (DLP), and heart disease. All comorbidity was defined as individuals with a history of the diagnosis indicated in the medical records of the patients plus having taken antihypertensive or antidiabetic or antidyslipidemic or heart medication within 2 weeks, respectively.

\subsubsection{Statistical Analysis}

The outcome of this study was the QOL of stroke patients. First, we carried out a descriptive analysis of the samples using percentage, mean, and SD.

Univariate and multiple linear regression analyses were performed using the following 4 models to estimate hazard ratios (HRs) and 95\% confidence intervals (95\% CI). Model 1 was constructed using clinical characteristics (NIHSS, BI, and $\mathrm{mRS}$ ) as independent variables and unadjusted by using univariate analysis was performed. Model 2 included age, sex, education, marital status, and occupation as independent variables in addition to clinical characteristics. Model 3 included comorbidity (HT, DM, DLP, and heart disease) in addition to clinical characteristics. Model 4 was all adjusted variables (clinical characteristics, age, sex, education, marital status, occupation, HT, DM, DLP, and heart disease). $P$-values of $<0.05$ were considered statistically significant. These statistical analyses were carried out by using SPSS Statistics version 26.0 (IBM Japan, Tokyo, Japan).

\subsubsection{Ethical Consideration}

Prior to the data collection, the study was approved by Institution Review Board (IRB) committee of the government hospital in Petchaburi province. Inform consent was performed and anonymity was ensured.

\section{Results}

A total of 113 stroke patients who met the inclusion criteria were recruited. The mean (SD) length of stay at the hospital was 7.53 (1.43) days. Descriptive data on clinical, sociodemographic, and comorbidity characteristics are presented in Table 1. The mean (SD; range) scores of the WHOQOL-BREF was 85.40 (10.47; 58 - 104).

The analysis of clinical characteristics associated with WHOQOL-BREF scores and simultaneously entered independent variables are summarized in Table 2. In the univariate analysis, model 1 found that lower NIHSS, higher BI score, lower mRS score, marital status (currently married), and employed status was independently associated with QOL. In addition, the clinical characteristics (NIHSS, $\mathrm{BI}$, and $\mathrm{mRS}$ ) were adjusted by sociodemographic characteristics in model 2 and adjusted by comorbidity in model 3 . The results found that higher BI and lower mRS scores were associated with QOL in all models after adjustment while 
Table 1. The basic characteristics of the participant $(n=113)$.

\begin{tabular}{|c|c|}
\hline Variables & Distribution in sample \\
\hline WHOQOL-BREF, mean (SD; range) scores & $84.30(13.21 ; 45-104)$ \\
\hline \multicolumn{2}{|l|}{ Clinical characteristics } \\
\hline NIHSS, mean (SD; range) scores & $4.00(4.90 ; 0-30)$ \\
\hline $\mathrm{BI}$, mean (SD; range) scores & $78.64(24.39 ; 20-100)$ \\
\hline mRS, mean (SD; range) scores & $2.47(1.49 ; 0-5)$ \\
\hline \multicolumn{2}{|l|}{ Socio-demographic characteristics } \\
\hline Age, mean (SD; range) years & $70.89(8.96 ; 60-92)$ \\
\hline Sex, male (\%):female (\%) & 73 (64.6): $40(35.4)$ \\
\hline \multicolumn{2}{|l|}{ Education, n (\%) } \\
\hline Uneducated & $25(22.1)$ \\
\hline Primary school & $79(69.9)$ \\
\hline Secondary school & $2(1.8)$ \\
\hline High school & $3(2.7)$ \\
\hline Diploma & $3(2.7)$ \\
\hline Bachelor's degree & $1(0.9)$ \\
\hline Master's degree and higher & $0(0)$ \\
\hline Marital status, unmarried (\%):married (\%) & $34(30.1): 79(69.9)$ \\
\hline Occupation, unemployed (\%):employed (\%) & $77(68.1): 36(31.9)$ \\
\hline \multicolumn{2}{|l|}{ Comorbidity } \\
\hline HT, no (\%):yes (\%) & $45(39.8): 68(60.2)$ \\
\hline DM, no (\%):yes (\%) & $81(71.7): 32(28.3)$ \\
\hline DLP, no (\%):yes (\%) & $86(76.1): 27(23.9)$ \\
\hline Heart disease, no (\%):yes (\%) & 107 (94.7):6 (5.3) \\
\hline
\end{tabular}

Abbreviation: $\mathrm{SD}=$ standard deviation, WHOQOL-BREF $=$ the abbreviated version of the World Health Organization Quality of Life, NIHSS = National Institute of Health Stroke Scale, BI = Barthel Index, mRS = Modified Rankin Scale, HT = hypertension, DM = diabetes mellitus, DLP = dyslipidemia.

NIHSS not associated with QOL. Moreover, in model 4 was all adjusted factors, high BI score was significantly different associated with QOL ( $\beta=0.312,95 \% \mathrm{CI}$ $=0.042,0.296, p$-value $=0.009)$, lower mRS score also was significantly different associated with QOL $(\beta=-0.371,95 \% \mathrm{CI}=-5.394,-1.162, p$-value $=0.003)$. Additional risk factor in this study was marital status (currently married) $(\beta=$ $0.155,95 \% \mathrm{CI}=0.226,8.666, p$-value $=0.039$ ).

\section{Discussion}

The principal findings of the present study indicated that the main effects of clinical outcomes on quality of life in older people with stroke at hospital discharge were Barthel index and modified Rankin scale which represented the stroke functional status and disability, respectively. The additional risk factor was marital status. 
Table 2. Multiple linear regression analyses of WHOQOL-BREF $(\mathrm{n}=113)$.

\begin{tabular}{ccccc}
\hline Variables & Model 1 & Model 2 & Model 3 & Model 4 \\
\cline { 2 - 4 } & $\boldsymbol{\beta}(95 \% \mathrm{CI})$ & $\boldsymbol{\beta}(95 \% \mathrm{CI})$ & $\boldsymbol{\beta}(95 \% \mathrm{CI})$ & $\boldsymbol{\beta}(95 \% \mathrm{CI})$ \\
\hline NIHSS, higher & $-0.516(-1.827,-0.958)^{* * *}$ & $-0.042(-0.591,0.366)$ & $-0.055(-0.657,0.362)$ & $-0.069(-0.692,0.318)$ \\
BI, higher & $0.690(0.300,0.448)^{* * *}$ & $0.328(0.054,0.302)^{* *}$ & $0.339(0.056,0.311)^{* *}$ & $0.312(0.042,0.296)^{* *}$ \\
mRS, higher & $-0.698(-7.360,-4.977)^{* * *}$ & $-0.382(-5.423,-1.335)^{* *}$ & $-0.379(-5.483,-1.218)^{* *}$ & $-0.371(-5.394,-1.162)^{* *}$ \\
Age, higher & $-0.147(-0.492,0.057)$ & $0.067(-0.128,0.326)$ & $0.091(-0.101,0.369)$ \\
Sex, male:female & $-0.080(-7.365,2.950)$ & $0.040(-2.887,5.067)$ & $0.042(-2.985,5.313)$ \\
Education, higher & $0.066(-1.884,3.917)$ & $-0.073(-3.248,0.996)$ & $-0.071(-3.284,1.075)$ \\
Marital status, & $0.238(1.585,12.065)^{*}$ & $0.145(0.039,8.259)^{*}$ & $0.155(0.226,8.666)^{*}$ \\
unmarried:married & & & $-0.052(-5.588,2.776)$ & $-0.050(-5.587,2.922)$ \\
Occupation, & $0.286(2.990,13.167)^{* *}$ & $0.135(-0.332,7.934)$ & $-0.037(-5.112,2.968)$ \\
unemployed:employed & $-0.075(-7.052,3.029)$ & & $-0.048(-5.501,2.684)$ & $0.131(-0.493,7.913)$ \\
HT, no:yes & $-0.111(-8.711,2.204)$ & & $0.013(-4.236,5.059)$ & $0.024(-3.999,5.457)$ \\
DM, no:yes & $-0.114(-9.275,2.255)$ & & $-0.079(-12.928,3.686)$ \\
DLP, no:yes & $-0.149(-19.678,2.145)$ & & & $-10.873,5.547)$ \\
Heart disease, no:yes & & & & $-0.045)$ \\
\hline
\end{tabular}

${ }^{\star} P<0.05{ }^{* *} P<0.01{ }^{* * *} P<0.001$. Model 1 was unadjusted. Model 2 was adjusted for clinical characteristics, age, sex, education, marital status, and occupation. Model 3 was adjusted for clinical characteristics, HT, DM, DLP, and heart disease. Model 4 was all adjusted (clinical characteristics, age, sex, education, marital status, occupantion, HT, DM, DLP, and heart disease). Abbreviation: CI = confidence interval, WHOQOL-BREF = the abbreviated version of the World Health Organization Quality of Life, NIHSS = National Institute of Health Stroke Scale, BI $=$ Barthel Index, $\mathrm{mRS}=$ Modified Rankin Scale, HT $=$ hypertension, $\mathrm{DM}=$ diabetes mellitus, $\mathrm{DLP}=$ dyslipidemia.

The activity of daily livings was an important factor which significantly associated with quality of life in older people with stroke. This is consistency with Mierlo and et al. [18] who studied quality of life in the Netherlands during the first two years after stroke and the results showed that patients with independent in ADL had higher scores on all 4 domains of QOL. In addition, Robinson-Smith, et al. [19] studied indicated that quality of life 6 months after stroke was related to the level of functional independence measured at discharge from the hospital. The ability to perform independent activities of daily living is closely linked with a rapid return to social life. Functional rehabilitation interventions for stroke patients with the limited ability of mobility and social cognition should focus on improving their abilities to perform independent daily living activities for improving their QOL and helping overcome physical, psychological, and social problems [20].

A study of Gunaydin and et al. [21] confirmed that the functional status during the assessment was the most relevant predictor of QOL. Improving physical function may help providing a better QOL for patients with stroke. The Barthel index has also been used in assessment for stroke patients, although the Barthel index is not multidimensional factorial relative to other QOL measures, higher Barthel index scores are an indicator of more social life involvement in that group. 
To improve the long-term QOL of the patients, a systematic clinical treatment and rehabilitation program in the stroke unit was carried out at acute stages. A psychological support team was heavily emphasized and worked closely with all patients to assess their abilities to improve health and enable them to return to an active life, given their impairment and disability [22].

In the present study, mRS score also indicated a significant differences association with QOL. These findings were consistent with Strege and et al. [23] who found that functional disability reported by mRS score at follow-up was significantly associated with a reduced model of both univariate and multivariate regression analysis model of QOL. In a cross-sectional study by Ali and et al. [24], mRS score captured more quality of life information than either NIHSS or BI scores. It was concluded that at 3 months after having stroke, mRS score was a useful indicator of overall health related QOL for stroke patients. Fischer and et al. [25] found that there were more impairments in QOL in stroke patients with a high mRS score and low BI score on both scales. For patients with worse mRS score, it was generally more likely that the patient could not walk around, had trouble taking care of himself/herself and doing usual activities. The contribution of mRS score as significant predictors of health related QOL was mostly explained by its association with the more objective dimensions (mobility, self-care, usual activities), and less likely to be explained by more subjective dimensions (pain/discomfort, anxiety/depression) [26].

The finding of the important relationship between marital status and quality of life in patients with stroke is consistent with the study of Osberg and et al. [27] and supports the current view that stable marital status is usually positively related to QOL [28]. However, married people are more satisfied than separated, divorced or widowed individuals, and slightly more satisfied than single people. This indicates that the emotional and social aspects of partner living are important for subjective well-being [29].

Disability and functional status, as measured by BI or mRS, were predictors of QOL for older people with stoke at hospital discharge. Carmo and et al. [30] defined disability as a dynamic process involving physical, mental, and emotional circumstances. Functional disability is understood as a part of this process and is considered an indicator of disability, assessed in daily activities through physical performance. In this clinical outcome study, both BI and mRS were associated with quality of life in older stoke people but not for NIHSS. Even patients have a severe stroke but functional recovery after the onset of stroke has shown by improving in BI and mRS scores. This will satisfy the patient by improving the independent ability in their daily lives for self-care.

The strength of this study was as follows. First, the diagnosis of stroke was followed $10^{\text {th }}$ revision of International Classification of Diseases (ICD-10) and confirmed by CT scan or MRI. Second, all comorbidity and clinical characteristics in this study were accurate because comorbidity diseases were diagnosed followed ICD-10 by the physician in a specific discipline, and measurement tools 
were standardized and validated. Third, collecting data through interviews allowed us to clarify questions or answers with the respondents, so we could be assured that all responses represented the perceptions of patients correctly.

However, various limitations have to mentioned as follows. First, the sample size of this study was small, which might cause some bias. Second, all patients were enrolled from a single hospital. A prospective multicenter clinical research was needed to validate our findings in order to investigate the relationship between improvements in stroke patients' QOL and clinical characteristics. Third, this was a cross-sectional analysis that did not provide information about changes over time in regard to QOL in stroke patients. Forth, not all patients were assessed at the same time after stroke, and the time interval between stroke and outcome evaluation was too broad. Lastly, selection bias might have caused underestimation of poor QOL in the study. Patients with severe stroke were unable to do self-report for their QOL, and we excluded those from the current study.

In conclusion, both disability and functional status were strongly affected by the QOL of older stroke people, including marital status was positively correlated with QOL at hospital discharge. The results of the present study were benefit for caregivers and healthcare providers for providing care of stroke patients after discharge from the hospital to prevent further decline in both clinical characteristics and QOL. Further longitudinal studies should be performed to assess the change of QOL over time.

\section{Conflicts of Interest}

The authors declare no conflicts of interest regarding the publication of this paper.

\section{References}

[1] GBD 2016 Stroke Collaborators (2019) Global, Regional, and National Burden of Stroke, 1990-2016: A Systematic Analysis for the Global Burden of Disease Study 2016. Lancet Neurology, 18, 439-458.

[2] Nilanont, Y., Nidhinandana, S., Suwanwela, N.C., Hanchaiphiboolkul, S., Pimpak, T., Tatsanavivat, P., et al. (2014) Quality of Acute Ischemic Stroke Care in Thailand: A Prospective Multicenter Country-Wide Cohort Study. Journal of Stroke \& Cerebrovascular Diseases, 23, 213-219. https://doi.org/10.1016/j.jstrokecerebrovasdis.2012.12.001

[3] Ahmadi, A., Tohidast, S.A., Mansuri, B., Kamali, M. and Krishnan, G. (2017) Acceptability, Reliability, and Validity of the Stroke and Aphasia Quality of Life Scale-39 (SAQOL-39) across Languages: A Systematic Review. Clinical Rehabilitation, 31, 1201-1214. https://doi.org/10.1177/0269215517690017

[4] Chabowski, M., Grzebien, A., Ziomek, A., Dorobisz, K., Lesniak, M. and Janczak, D. (2017) Quality of Life after Carotid Endarterectomy: A Review of the Literature. Acta Neurologica Belgica, 117, 829-835. https://doi.org/10.1007/s13760-017-0811-x

[5] Sturm, J.W., Donnan, G.A., Dewey, H.M., Macdonell, R.A., Gilligan, A.K., Srikanth, V., et al. (2004) Quality of Life after Stroke: The North East Melbourne Stroke Incidence Study (Nemesis). Stroke, 35, 2340-2345.

https://doi.org/10.1161/01.STR.0000141977.18520.3b 
[6] Haley, W.E., Roth, D.L., Kissela, B., Perkins, M. and Howard, G. (2011) Quality of Life after Stroke: A Prospective Longitudinal Study. Quality of Life Research, 20, 799-806. https://doi.org/10.1007/s11136-010-9810-6

[7] Ramos-Lima, M.J., Brasileiro, I.C., Lima, T.L. and Braga-Neto, P. (2018) Quality of Life after Stroke: Impact of Clinical and Sociodemographic Factors. Clinics, 73, e418. https://doi.org/10.6061/clinics/2017/e418

[8] Vercelli, S., Ferriero, G., Bravini, E., Al Yazeedi, W., Salgovic, L., Caligari, M., et al. (2017) A Simple Orthosis Solves a Problem in a Patient with a Dystonic Finger after Stroke. Journal of Hand Therapy, 30, 113-115. https://doi.org/10.1016/j.jht.2016.04.003

[9] Rangel, E.S., Sliva Belasco, A.G. and Dicinni, S. (2013) Qualidade de Vida de Pacientes com Acidente Vascular Cerebral em Reabilitação. Acta Paulista de Enfermagem, 26, 205-212. https://doi.org/10.1590/S0103-21002013000200016

[10] Froes, K.S., Valdes, M.T., Lopes, D.P. and Silva, C.E. (2011) Factors Associated with Health-Related Quality of Life for Adults with Stroke Sequelae. Arquivos de Neuro-Psiquiatria, 69, 371-376. https://doi.org/10.1590/S0004-282X2011000300020

[11] Butsing, N., Tipayamongkholgul, M., Ratanakorn, D., Suwannapong, N. and Bundhamcharoen, K. (2019) Social Support, Functional Outcome and Quality of Life among Stroke Survivors in an Urban Area. Journal Pacific Rim Psychology, 13, e4. https://doi.org/10.1017/prp.2019.2

[12] Chen, Q., Cao, C., Gong, L. and Zhang, Y. (2019) Health Related Quality of Life in Stroke Patients and Risk Factors Associated with Patients for Return to Work. Medicine, 98, e15130. https://doi.org/10.1097/MD.0000000000015130

[13] Nicholas-Larsen, D.S., Clark, P.C., Zeringue, A., Greenspan, A. and Blanton, S. (2005) Factors Influencing Stroke Survivors' Quality of Life during Subacute Recovery. Stroke, 36, 1480-1484. https://doi.org/10.1161/01.STR.0000170706.13595.4f

[14] Mahatnirunkul, S., Tuntipivatanaskul, W. and Pumpisanchai, W. (1998) Comparison of the Whoqol-100 and the Whoqol-Bref (26 Items). Journal of Mental Health of Thailand, 5, 4-15.

[15] Wityk, R.J., Pessin, M.S., Kaplan, R.F. and Caplan, L.R. (1994) Serial Assessment of Acute Stroke Using the NIH Stroke Scale. Stroke, 25, 362-365. https://doi.org/10.1161/01.STR.25.2.362

[16] Mahoney, F.I. and Barthel, D.W. (1965) Functional Evaluation: The Barthel Index. Maryland State. Maryland Medical Journal, 14, 56-61.

[17] Duncan, P.W., Jorgensen, H.S. and Wade, D.T. (2000) Outcome Measures in Acute Stroke Trials: A Systematic Review and Some Recommendations to Improve Practice. Stroke, 31, 1429-1438. https://doi.org/10.1161/01.STR.31.6.1429

[18] Mierlo, M.L., Van Heugten, C.M., Post, M.W., Hajos, T.R., Kappelle, L.J. and Visser-Meily, J.M. (2016) Quality of Life during the First Two Years Post Stroke: The Restore4Stroke Cohort Study. Cerebrovascular Diseases, 41, 19-26. https://doi.org/10.1159/000441197

[19] Robinson-Smith, G., Johnson, M.V. and Allen, J. (2000) Self-Care, Self-Efficacy, Quality of Life, and Depression after Stroke. Archives of Physical Medicine and Rehabilitation, 81, 460-464. https://doi.org/10.1053/mr.2000.3863

[20] Kim, K., Kim, Y.M. and Kim, E.K. (2014) Correlation between the Activities of Daily Living of Stroke Patients in a Community Setting and Their Quality of Life. Journal of Physical Therapy Science, 26, 417-419. 
[21] Gunaydin, R., Karatepe, A.G., Kaya, T. and Ulutas, O. (2011) Determinants of Quality of Life (QoL) in Elderly Stroke Patients: A Short-Term Follow-Up Study. Archives of Gerontology and Geriatrics, 53, 19-23. https://doi.org/10.1016/j.archger.2010.06.004

[22] Mudaliar, M.R., Yiragamreddy, S.R., Tejashwani, P.P., Umapathi, S., Sake, N. and Sharma, S. (2018) Quality of Life in Stroke Patients Using SSQoL Scale and Barthel Index. Indian Journal of Pharmacy Practice, 11, 44-50.

[23] Strege, R.J., Kiefer, R. and Herrmann, M. (2019) Contributing Factors to Quality of Life after Vertebral Artery Dissection: A Prospective Comparative Study. BMC Neurology, 19, 312-317.

[24] Ali, M., Fulton, R., Quinn, T., Brady, M. and Collaboration, V. (2013) How Well Do Standard Stroke Outcome Measures Reflect Quality of Life? A Retrospective Analysis of Clinical Trial Data. Stroke, 44, 3161-3165. https://doi.org/10.1161/STROKEAHA.113.001126

[25] Fischer, U., Anca, D., Arnold, M., Nedeltchev, K., Kappeler, L., Ballinari, P., et al. (2008) Quality of Life in Stroke Survivors after Local Intra-Arterial Thrombolysis. Cerebrovascular Diseases, 25, 438-444. https://doi.org/10.1159/000126917

[26] Shing, Y.Y. (2016) Health-Related Quality of Life in Singapore Stroke Patients and Its Association with Clinical Outcomes. https://scholarbank.nus.edu.sg/handle/10635/129329

[27] Osberg, J.S., Dejong, G., Haley, S., Seward, M.L., Mcginnis, M.P. and Germaine, J. (1998) Predicting Long-Term Outcome among Post-Rehabilitation Stroke Patients. American Journal of Physical Medicine \& Rehabilitation, 67, 94-103. https://doi.org/10.1097/00002060-198806000-00002

[28] Kim, P., Warren, S., Madill, H. and Hadley, M. (1999) Quality of Life of Stroke Survivors. Quality of Life Research, 8, 293-301. https://doi.org/10.1023/A:1008927431300

[29] Yeoh, Y.S., Koh, G.C.H., Tan, C.S., Tu, T.M., Singh, R., Chang, H.M., et al. (2019) Health-Related Quality of Life Loss Associated with First-Time Stroke. PloS ONE, 14, e0211493. https://doi.org/10.1371/journal.pone.0211493

[30] Carmo, J.F., Morelato, R.L., Pinto, H.P. and Oliveira, E.R.A. (2015) Disability after Stroke: A Systematic Review. Fisioterapia em Movimento, 28, 407-418. https://doi.org/10.1590/0103-5150.028.002.AR02 